\title{
BRITISH ASSOCIATION OF PAEDIATRIC SURGEONS
}

The ninth Annual Meeting of the British Association of Paediatric Surgeons was held in London on September 4, 5 and 6, 1962, under the Presidency of Mr. R. B. Zachary. It was attended by 145 members and approximately 100 guests representing 29 countries.

The scientific meeting opened with a symposium on 'Small Bowel Obstruction in Childhood', on the Tuesday morning, September 4, and in the afternoon scientific films and papers on various subjects were presented. Dr. E. B. D. Neuhauser, of the Boston Children's Medical Centre, gave a lecture by invitation of the British Association of Paediatric Surgeons and the Faculty of Radiology, at the Royal College of Surgeons.

On the Wednesday morning, September 5, there was a symposium on Exomphalos, and in the afternoon the Association was invited by the late Professor Ian Aird to attend lectures and demonstrations at the Postgraduate Medical School, Hammersmith. In the evening Oxygenaire Ltd. invited the Association to a Buffet Supper and a demonstration of recent advances of oxygen therapy in infancy.

On the Thursday morning, September 6, papers were given on various subjects, and during the afternoon there were clinical demonstrations by the surgeons and a pathological demonstration by Dr. M. Bodian at The Hospital for Sick Children, Great Ormond Street. Later on in the afternoon members of the Association attended the Simpson Smith Memorial Lecture on Skeletal Deformities by Dr. M. Sulamaa, of Helsinki.

At the Annual Dinner the guest of honour was Sir George Godber, Principal Medical Officer at the Ministry of Health.

The tenth Annual meeting will be held in Sheffield from July 9 to 11,1963 .

The following are abstracts of papers read at the meeting in Stockholm and not published in full:

Denis Browne (London). 'Spina Bifida with Narrow Dural Pedicle.' This paper treats of one particular form of spina bifida which has a forbidding appearance but is easy to treat by very simple means that give satisfactory results. In it there may be a very large external tumour due to the tendency, unique in the body, of these spinal deformities to form large amounts of primitive unorganized tissue. There is, however, little or no paralysis or absence of sensation below the lesion, and this is a certain sign that however large the dural sac inside the tumour, there will be a narrow pedicle connecting it to the spinal tube of dura.

The treatment recommended is to expose this pedicle through a transverse elliptical incision, free it from any connexions to the bony opening through which it emerges, ligature it with a non-absorbable thread, and cut off the whole mass distal to the ligature. No attempt is made to dissect out fibres resembling nerves, or to close the gap in the bone by means of flaps of fascia.

The arguments against this crude and simple proceeding are: (1) That the sac is an absorbing mechanism for the cerebrospinal fluid, and that its removal may in consequence cause the onset of hydrocephalus. There is no doubt that hydrocephalus may be started or accelerated by operations on spina bifida tumours, but the mechanism here is the descent of the brain-stem into the foramen magnum permitted by the release of pressure below it, so plugging the circulation of the fluid. The vast majority, if not all, of such cases already have an Arnold-Chiari deformity, and none of them are of the type described.

(2) That functioning nerves may be present in the tissues removed. There are almost always fibres running inside the dural sac, which have a superficial resemblance to true nerves, but on stimulation no action on the muscles below is seen, and neither microscopical section nor the most careful dissection suggest that these strands have any function. The real test is the absence of any bad effects from the drastic removal of the whole sac.

Finally, it is maintained that more complicated methods of treatment, including the preservation of parts or the whole of the dural sac may do more harm than good owing to pressure on the true spinal nerves when this mass of tissue is forced down into the small bony gap. Liability to these pressure effects would be greater if flaps of dura were to be sutured over it.

O. Knutrud (Oslo, Norway). 'The Post-traumatic Metabolic Response in the Newborn with Special Reference to Potassium, Magnesium and Phosphorus Metabolism.' Post-operative metabolic studies have been performed on 28 newborn babies and eight normal controls. It is clearly shown in these investigations that potassium is lost in excess after trauma in the newborn babies. Magnesium, however, is lost in accordance with nitrogen, demonstrating an identical $\mathrm{Mg} / \mathrm{N}$ ratio as found in lean muscle tissue. Phosphorus is found to be lost in excess of nitrogen and the $P / N$ ratio is following the same pattern as the $K / N$ ratio.

The early excessive potassium and phosphorus losses are calculated and the ratio between them is found to be the same as it is within the cell in the phosphate-carbohydrate mono-esters at a $p \mathrm{H}$ of $7 \cdot 1$. This indicates that the excessive post-traumatic potassium loss is secondary to an early release of the intracellular phosphorus-containing carbohydrate intermediates-and it is suggested that this is a reasonable explanation of the post-traumatic potassium shift. 
ERNST RÜTHER (Bremen). 'Hydrometrocolpos in Newborn Infants with Gynatresia.' The child converts all placentogenic oestrogens into oestriol. In the last few months of foetal life, the high blood oestriol levels cause marked proliferation of the vaginal epithelium and the endocervix. If an anatomical anomaly prevents cervical discharge, the mucus accumulates. The most frequent form of anomaly is atresia of the vagina behind the hymenal ring. Occlusions higher up in the cervix can occur in dual formations of the internal genitals.

The mildest form we saw was in children with intersex genitals. The vagina was not closed but discharge was functionally inhibited; this may give rise to relapsing pyocolpos.

In anal atresia the opening of the rectum into the cloaca may be so near the cervix that the uterus becomes distended with meconium. We have only seen this once and puncture of the retention tumour on laparotomy yielded $200 \mathrm{ml}$. meconium.

Ascending infection with fever can occur if the urogenital sinus forms a functional stenosis. During a pull-through procedure for anal atresia, we found that the uterine cavity, which had previously only contained mucus, was severely infected and contaminated with intestinal contents.

After the first or second week of life no further increase of the accumulation need be feared. An increase in size can be caused by ascending infection.

In most cases laparotomy should be done, particularly in order to clarify the anatomical situation. From the abdomen, puncture or evacuation after incision is sufficient. The secretion may be very viscid.
It is unpleasant to have to undertake surgery on a newborn baby in anticipation of disturbances that may occur at menstruation. But the parents should be told that before puberty, when the oestrogens increase, the congestion will recur and correction must be provided for in time.

Herbert B. Eckstein (London). 'Exomphalos: a Review of 100 Cases.' The history of exomphalos was briefly reviewed. A series of 100 cases seen at Great Ormond Street between 1938 and 1961 was presented.

The overall mortality was $44 \%$ and it was shown that of all factors affecting the mortality the association of other congenital abnormalities was the most important. Only one baby weighing less than $5 \mathrm{lb}$. survived and only those of $7 \mathrm{lb}$. or more have a good prognosis. Postnatal rupture of the sac and the size of the defect do not affect the mortality greatly, but all cases with definite antenatal rupture died.

There was a group of cases, accounting for $10 \%$ in this series, in which the cord was cranial to the defect in the abdominal wall; ectopia vesicae and hind-gut agenesis were frequent findings in this group, but cardiac abnormalities were met with only in cases where the cord was caudal to the defect.

The various forms of treatment used in the series were presented and it is thought that 11 cases may have died of too tight a closure. Post-operative respirator therapy is advocated in suitable cases.

Whereas survivors with small defects develop into children of normal size, those with large defects tend, as a group, to remain small. 